Proceedings of the International School and Conference on Optics and Optical Materials, ISCOM07, Belgrade, Serbia, September 3-7, 2007

\title{
Negative Refraction as a Source of Some Pedagogical Problems
}

\author{
V.G. VESELAGO*
}

Moscow Institute of Physics and Technology

9 Institutskii per., Dolgoprudny, 141700 Moscow Region, Russia 2

A.M. Prokhorov Institute of General Physics, RAS, Russia

38 Vavilov str., 119991, Moscow, Russia

The appearance of material with negative values of dielectric and magnetic permeabilities $\varepsilon$ and $\mu$ and with negative index of refraction $n$ forces to revise the accustomed forms of some well-known formulae of electrodynamics. This is connected with fact, that these correlations in repressing majority of sources is written in tacit suggestion that magnetic permeability of most of materials is $\mu=1$, factor of refraction $n=\varepsilon$ and is undoubtedly executed the correlation $n>0$. Such approach could be named as "nonmagnetic". However, if $n<0$, this nonmagnetic approach can bring very hard mistakes.

PACS numbers: 78.20.Ci, 41.20.Jb

\section{Introduction}

Introduction to broad scientific circulation the words "metamaterials", "negative refraction", "negative index of refraction", "lefthanded materials" and other terms derived by afore-cited, has brought some unexpected consequences for strategy of teaching of optics, electrodynamics and related disciplines. This fact is bound first of all with the fact that before recent time a index of refraction for isotropic materials always was taken as positive value, equal to

$$
n=\sqrt{\varepsilon \mu} .
$$

In this relation electric permeability $\varepsilon$ and magnetic permeability $\mu$ were also considered as positive values. The recognition of fact that $n$, and $\varepsilon$ with $\mu$, can be, in principle, simultaneously negative values, generates very simple question — in what measure remain equitable many formulae, determinations and correlations, in which these values enter if they are negative. Therefore, obviously that well-

*e-mail: v.veselago@relcom.ru 
-known formula for Snell law

$$
\frac{\sin \varphi}{\sin \phi}=n
$$

will be valid for negative values of $n$, but, of course, it is necessary to define correctly negative angles, as it is shown in Fig. 1.

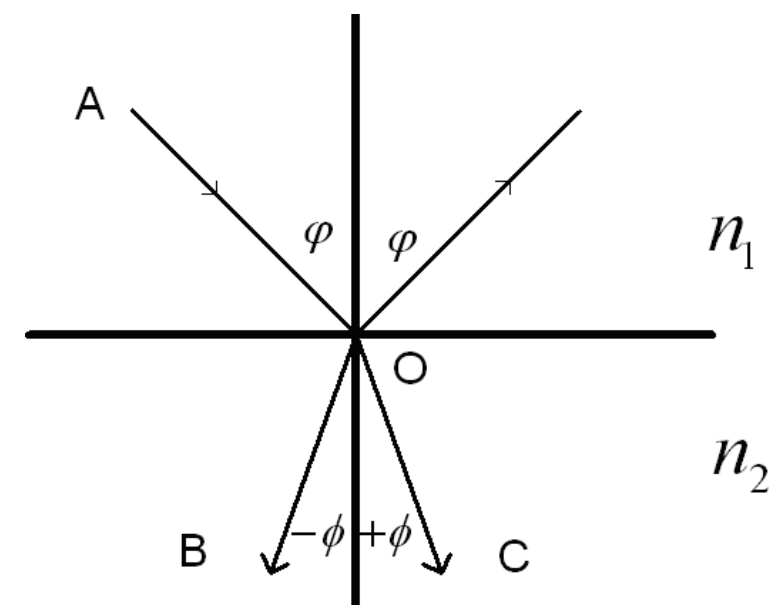

Fig. 1. Refraction of beam of light on interface between two isotropic media. In every case $\sin \varphi / \sin \phi=n_{1} / n_{2}=n$, but if $n$ is positive, the beam path is $A O C$, but for negative $n$ the path is $A O B$.

In the same way, as for Snell law, the equitable well-known formulae for the Doppler and Cherenkov effects will remain when we change $+n \rightarrow-n$.

However, situation sharply changes if we consider, for example, the commonly used Fresnel formulae for material with negative refraction. Therefore, following most of textbooks, reflection coefficient for wave with perpendicular polarization is

$$
R_{\perp}=\frac{n_{1} \cos \varphi-n_{2} \cos \phi}{n_{1} \cos \varphi+n_{2} \cos \phi} .
$$

It is clear that substituting negative $n_{1}$ and $n_{2}$ in this formula can give undoubtedly wrong value for $R_{\perp}$. Really correct formula for $R_{\perp}$ has the form

$$
R_{\perp}=\frac{z_{2} \cos \varphi-z_{1} \cos \phi}{z_{2} \cos \varphi+z_{1} \cos \phi}
$$

Here values $z_{1}$ and $z_{2}$ are so-called wave impedances of two media, and are, accordingly,

$$
z_{1,2}=\sqrt{\mu_{1,2} / \varepsilon_{1,2}}
$$

Not difficult to see that formula (4) is equivalent with formula (3) in the case when

$$
\mu_{1}=\mu_{2}=1
$$

and, hereunder, 


$$
z=\sqrt{1 / \varepsilon}=1 / n
$$

Equation (6) is a condition of so-called "nonmagnetic approach", which implies that we work with nonmagnetic materials, whose magnetic permeability is identically unity, and for which correlation (7) is due to (5). Such approach undoubtedly is ineffective for metamaterials in general, and, in particular, to metamaterials with negative refraction, whose magnetic permeability can be not only different from unity, but even negative.

Acceptance of the identity $\mu=1$ is equivalent to acceptance of equality of vectors of magnetic field $\boldsymbol{H}$ and magnetic induction $\boldsymbol{B}$ :

$$
\boldsymbol{B}=\boldsymbol{H}
$$

and so except term "nonmagnetic approach" such approach can be named as "three-vector approach". Exactly such approach presents in most textbooks and scientific literature, like, for example, in the book under Landau and Lifshitz. As a rule, in such literature presents a phrase like "since magnetic permeability of all known material on enough high frequency is unity, we shall suppose that vectors $H$ and $B$ of these materials are equal". Naturally, such approach does not leave any place for introduction of wave resistance $z$. Hereunder this approach brings rough physical mistakes, for example, very famous statement that condition of absence reflections of wave on the interface between two media is

$$
n_{1}=n_{2} .
$$

Really this condition must be recorded as equality of two impedances

$$
z_{1}=z_{2} \text {. }
$$

List of some most widely-used formulae written in nonmagnetic approach and in correct form is placed in Table.

TABLE

Some formulae of electrodynamics, written in nonmagnetic and correct forms.

\begin{tabular}{c|c|c}
\hline \hline Physical law & $\begin{array}{c}\text { Equation for nonmagnetic } \\
\text { approach }\end{array}$ & Correct equation \\
\hline $\begin{array}{c}\text { Snellius, Doppler, Cherenkov } \\
n=\sqrt{\varepsilon} \rightarrow n=\sqrt{\varepsilon \mu} \\
\text { if } \varepsilon, \mu<0 \text { then } n<0\end{array}$ & $\sin \varphi / \sin \psi=n_{21}=\sqrt{\varepsilon_{2} / \varepsilon_{1}}$ & $\begin{array}{c}\sin \varphi / \sin \psi=n_{21} \\
\sqrt{\varepsilon_{2} \mu_{2} / \varepsilon_{1} \mu_{1}}\end{array}$ \\
\hline $\begin{array}{c}\text { Fresnel } \\
n=\sqrt{\varepsilon} \rightarrow 1 / z=\sqrt{\varepsilon / \mu}\end{array}$ & $r_{\perp}=\frac{n_{1} \cos \varphi-n_{2} \cos \psi}{n_{1} \cos \varphi+n_{2} \cos \psi}$ & $r_{\perp}=\frac{z_{2} \cos \varphi-z_{1} \cos \psi}{z_{2} \cos \varphi+z_{1} \cos \psi}$ \\
\hline $\begin{array}{c}\text { reflection coefficient for } \\
\text { normal fall of light on the } \\
\text { border between two media }\end{array}$ & $r=\left(n_{1}-n_{2}\right) /\left(n_{1}+n_{2}\right)$ & $r=\left(z_{2}-z_{1}\right)\left(z_{2}+z_{1}\right)$ \\
\hline condition for full matching & $n_{1}=n_{2}$ & $z_{1}=z_{2}$ \\
\hline Brewster angle & $\tan \varphi=n$ & $\tan \varphi=\sqrt{\frac{\varepsilon_{2}}{\varepsilon_{1}} \frac{\varepsilon_{2} \mu_{1}-\varepsilon_{1} \mu_{2}}{\varepsilon_{2} \mu_{2}-\varepsilon_{1} \mu_{1}}}$
\end{tabular}


The motivation of nonmagnetic approach usually comes from the fact that in optical domain magnetic permeability is a unity. This is really so for materials, whose magnetic characteristics are defined by small particles with atomic sizes. These materials often have a name of so-called "natural" materials, for example like crystals and liquids. However situation greatly differs for the case of "artificial" materials, to which usually refer metamaterials. The magnetic characteristics of these materials are defined by the currents on conducting, metallic elements of these materials. Often this fact is a reason for considering of metamaterials as the media without definite two permeabilities $\varepsilon$ and $\mu$. Indeed, difference between socalled natural material and metamaterials is quantitative, rather than principal. Really, one or another material could be described by means of electric or magnetic permeability; it is necessary that wavelength of radiation will be much more than typical size of elements, forming material and distances between these elements. Therefore if we consider that lattice constant $\delta$ of majority of natural materials is close to value $5 \times 10^{-5} \mu \mathrm{m}$, but in optical domain a wavelength $\lambda$ is close to $0.5 \mu \mathrm{m}$, that quotient $\lambda / \delta$ for these values turns out to be close to $10^{4}$. In first experiments on observation of passing of waves of microwave range through metamaterials this quotient there was about 10 . Coefficient $\lambda / \delta$ has approximately such value in experiments with metamaterials in optical range. Naturally, this value of $\lambda / \delta$ is noticeably less than quotient for "natural" material in optical range, but however quotient $\lambda / \delta \approx 10$ is wholly enough for use approach, based on electric and magnetic permeability. Exactly so contraposition "natural" and "artificial" material is deprived in the physical sense.

Thereby, we see that appearance of metamaterials requires contributing the essential changes to strategy of teaching of electrodynamics and physics. The corresponding courses must begin with consequent introduction of four vectors $E$, $D, H, B$, and obligatory introduction of the values of wave impedance $z=\sqrt{\mu / \varepsilon}$ of media, and only afterwards possible come to three-vector approach if for one or another media magnetic permeability turns out to be identically equal to unity, and, accordingly, turns out to be the equitable correlation (8). Herewith in any case it is impossible to abandon notions of wave impedance. It is necessary to take into account that even $\mu \equiv 1$ and, consequently, equation

$$
z=\sqrt{1 / \varepsilon}=1 / n
$$

is valid, so values $z$ and $n$ become interdependent, their physical sense remains absolutely different. It is necessary to remember that wave impedance for vacuum, equal to $z_{0}=377 \Omega$, is a world constant, exactly in the same way as speed of light in vacuum $c=3 \times 10^{10} \mathrm{~cm} / \mathrm{s}$. Therefore, electromagnetic characteristics of material are wholly possible to be described not on language " $\varepsilon$ and $\mu$ " but also on language " $n$ and $z$ ", using correlations

$$
\varepsilon=n / z
$$

and 


$$
\mu=n z .
$$

However under such approach it is easy to make a mistake, considering that under negative $\varepsilon$ and $\mu$ value $n$ is positive, but $z$ - negative. Really, the value $z$ always must be taken positive, outside of dependences on sign of $\varepsilon$ and $\mu$. This follows from the fact that value $z$ defines the attitude of amplitudes of vectors $H$ and $E$ in wave, which does not depend on signs of $\varepsilon$ and $\mu$. In this it is easy to make sure, having considered boundary conditions for perpendicular incidence of wave on interface between two media, if one of them is righthanded, but another - lefthanded. Under such turning the fields $H$ and $E$ are not changed, and, consequently value $z$ is not changed, equal to

$$
z=H / E \text {. }
$$

Another approach to this problem is possible, too.

Let us write value $n$ for the case of negative $\varepsilon$ and $\mu$ :

$$
n=\sqrt{\varepsilon \mu}=\sqrt{\varepsilon} \sqrt{\mu}=\mathrm{i} \sqrt{|\varepsilon|} \mathrm{i} \sqrt{|\mu|}=-\sqrt{|\varepsilon|} \sqrt{|\mu|}<0 \text {. }
$$

In this case we get undoubtedly negative value of $n$.

Similar calculation gives for $z$ under negative values of $\varepsilon$ and $\mu$ an opposite, undoubtedly positive result

$$
z=\frac{\sqrt{\mu}}{\sqrt{\varepsilon}}=\frac{\sqrt{-|\mu|}}{\sqrt{-|\varepsilon|}}=\frac{\mathrm{i} \sqrt{|\mu|}}{\mathrm{i} \sqrt{|\varepsilon|}}=\frac{\sqrt{|\mu|}}{\sqrt{|\varepsilon|}}>0 .
$$

Therefore, it is clear that wave impedance $z$ is positive, independently of signs of $n$, $\varepsilon$, and $\mu$. It is very important that some formulae for media with arbitrary values of $\varepsilon$ and $\mu$ are changed very strong, like, for example formula for the Brewster angle (see last row in Table).

All these considerations are necessary to be taken into account during process of studying optics and electrodynamics.

\section{Acknowledgments}

This work is supported by the Russian Foundation of Basic Research, grant \#07-02-00233. 